\title{
Quantitative Evaluation of Pubic Symphysis in Late Pregnancy Using
}

\section{T2* Mapping}

\author{
Xia Zhang ${ }^{1,2}$, Tao Gong ${ }^{1,3}$, Shuhui Yuan ${ }^{4}$ and Guangbin Wang ${ }^{1, *}$ \\ ${ }^{1}$ Shandong Medical Imaging Research Institute, Shandong University, Jinan, P.R. China \\ ${ }^{2}$ Yiyuan People's Hospital, Zibo, P.R. China \\ ${ }^{3}$ Department of Radiology, Shandong Provincal Hospital Affliated to Shandong First Medical University, Jinan, P.R. China \\ ${ }^{4}$ Jinan Blood Center, Jinan, P.R. China \\ "Corresponding author: Shandong Medical Imaging Research Institute, Shandong University, Jinan, P.R. China. Email: wgb7932596@hotmail.com
}

Received 2020 August 01; Revised 2020 September 06; Accepted 2021 February 20.

\begin{abstract}
Background: $2^{*}$ mapping is a reliable tool in articular cartilage imaging. It has been widely used to evaluate the degeneration of knee joint and intervertebral discs. Degenerative joint disease of pubic symphysis is the primary cause of groin pain during late pregnancy.

Objectives: To evaluate the alterations of pubic symphyseal cartilage during late pregnancy using T2* mapping.

Patients and Methods: Sixty-one first-pregnancy women, 48 second-pregnancy women and 64 nulliparous women were enrolled in this case-control study. All subjects underwent MRI exam of the pubic symphysis on a $1.5 \mathrm{~T}$ MR scanner, including T2* mapping sequence. Regions of interest (ROIs) were hand-drawn on $\mathrm{T}^{*}$ maps along the left and right side of the cartilage and anterior/middle/posterior sub-regions of each side. T2* values were analyzed and compared among the three groups using analysis of variance. The average pubic symphyseal width was measured. The correlation between pubic symphyseal width and T2* values of cartilage were analyzed using Pearson's correlation coefficient.

Results: Compared with nulliparous women, $\mathrm{T} 2 *$ values increased significantly in late pregnancy in the left/right cartilage of pubic symphysis. The differences were mainly driven by the posterior sub-region; while, no differences were found between women during first and second pregnancies. The positive linear correlation between symphyseal width and mean $\mathrm{T} 2{ }^{*}$ values of the cartilage was only seen in the group of women during first pregnancy, with no significant linear correlation in the other two groups.

Conclusion: $\mathrm{T}^{*}$ mapping is a sensitive quantitative method capable of detecting changes in the pubic symphyseal cartilage related to pregnancy, and the $\mathrm{T} 2 *$ values increased significantly during late pregnancy.
\end{abstract}

Keywords: Pubic Symphysis, Magnetic Resonance Imaging, Pregnancy, Cartilage

\section{Background}

The pubic symphysis is a secondary cartilaginous joint located between the left and right pubic bone, covered by a thin layer of hyaline cartilage attached to the fibrocartilage (1). Degeneration joint disease of the pubic symphysis, which can cause groin pain, always accompanies with aging and postpartum, and it is very common in pregnant women, such as symphysis pubis dysfunction.

$\mathrm{T} 2$ relaxation times can be used to measure cartilage degeneration due to their dependency on the water and collagen content of the extracellular matrix as well as orientation of collagen fibers (2). T2 mapping has been used in multiple clinical studies mostly in the knee (2-7), hip (4, 8,9 ) and spine (10-16), many of which demonstrated that T2 mapping was able to identify early cartilage degeneration.

The $\mathrm{T}^{*}$ value is related to $\mathrm{T} 2$ as follows: $1 / \mathrm{T} 2 *=1 / \mathrm{T} 2+$
1/T2', where $1 / \mathrm{T} 2$ ' can be given by $\gamma \Delta \mathrm{B}$. So $\mathrm{T} 2 *$ values are also influenced by local susceptibility fields, $\mathrm{T} 2 *$ mapping is potential to provide greater sensitivity to the injury of the calcified cartilage zone (17), such as pregnancy-related degeneration of the pubic symphyseal cartilage. $\mathrm{T2}$ * mapping provides information on spatial macromolecule architecture and its interaction with water molecule mobility, could also be used to detect early abnormalities in the articular cartilage $(18,19)$. Compared with traditional T2 mapping, T2* mapping could bring added benefits of shorter acquisition time, high signal-to-noise ratio, and three-dimensionality $(17,20)$, which makes it be more suitable for pregnant women.

Literature search showed no studies with T2* mapping for evaluation of the pubic symphysis during pregnancy. The hormone called relaxin released during pregnancy 
increases the elasticity of tendons, ligaments, and muscles, widen the pubic width, and stretch the cartilage between the two pubic bones for the demand of child-birth. When the changes of ligaments and cartilage go beyond the physiological limit, the pregnant woman suffers from groin pain. Normal T2* values of cartilage between the pubic bones in pregnant women are useful for determining pathologic changes.

\section{Objectives}

The purpose of this study was to evaluate the cartilage of pubic symphysis alterations during late pregnancy using T2* mapping.

\section{Patients and Methods}

\subsection{Human Subjects}

This case-control study was approved by the Ethical Committee of Shandong Provincial Hospital, and all the participants provided written informed consent for this study. Subjects enrolled in this study were all Han Chinese, the inclusion criteria was as follows: (1) Healthy nulliparous women at child-bearing age without any injury to the pelvis; (2) healthy first- and second-pregnant women at late pregnancy (28 weeks until birth) (usually between 37 and 42 weeks of pregnancy), and none of the subjects complained of pubic pain during their pregnancies. We excluded the subjects who could not endure magnetic resonance imaging (MRI) examination because of claustrophobia or other reasons. MRI data with too much artifact was also abandoned.

\subsection{Magnetic Resonance Acquisition}

All participants underwent MRI of the pubic symphysis on a 1.5T MAGNETOM Amira (Siemens Shenzhen Magnetic Resonance Ltd., China) with body and spine array coils. The subjects were all lying in a supine position with mild hip flexion during the MRI examination.

The MRI protocols included a multi-echo gradient recalled echo sequence with five consecutive echoes for T2* mapping. The parameters were as follows: repetition time (TR) $423 \mathrm{~ms}$, echo time (TE) 4.35, 11.83, 19.31, 26.79, and 34.27 ms, flip angle $60^{\circ}$, bandwidth $260 \mathrm{~Hz} /$ pixel, field of view $320 \times 320 \mathrm{~mm}^{2}$, matrix: $256 \times 256,11$ slices, slice thickness $3.0 \mathrm{~mm}$, voxel size $1.25 \times 1.25 \times 3.0 \mathrm{~mm}^{3}$, and acquisition time was 3:13 min.

\section{3. $22^{*}$ Mapping Processing and Quantification}

The $\mathrm{T}^{*}$ maps were generated using an inline processing package (Syngo MapIt; Siemens Healthcare, Erlangen, Germany), which used a log-linear, least squares method to fit the echo intensities.

The slice with the narrowest pubic symphyseal width at the middle part was identified, the average pubic symphyseal width and anterior pubic ligament at mid-point were then measured on $\mathrm{TE}=11.83 \mathrm{~ms}$ images (Figure 1 ). The regions of interest (ROIs) analysis were undertaken manually by one senior radiologist. Two ROIs were hand drawn on $\mathrm{TE}=11.83 \mathrm{~ms}$ images of the left and right side of the cartilage, and three sub-regions (anterior, middle and posterior) were drawn on each side of the cartilage (Figure 2) for zonal evaluation. The ROIs were positioned within the fibrocartilage zone seen in Figure 2. The volume for left/right region was $28.6 \pm 4.5 \mathrm{~mm}^{2}$; and the sub-region was $7.0 \pm$ $0.8 \mathrm{~mm}^{2}$. Then the same ROIs were copied to the T2* maps to obtain $\mathrm{T} 2 *$ values. Mean values for $\mathrm{T} 2 *$ were used for statistical analysis and expressed as mean \pm standard deviation (SD).

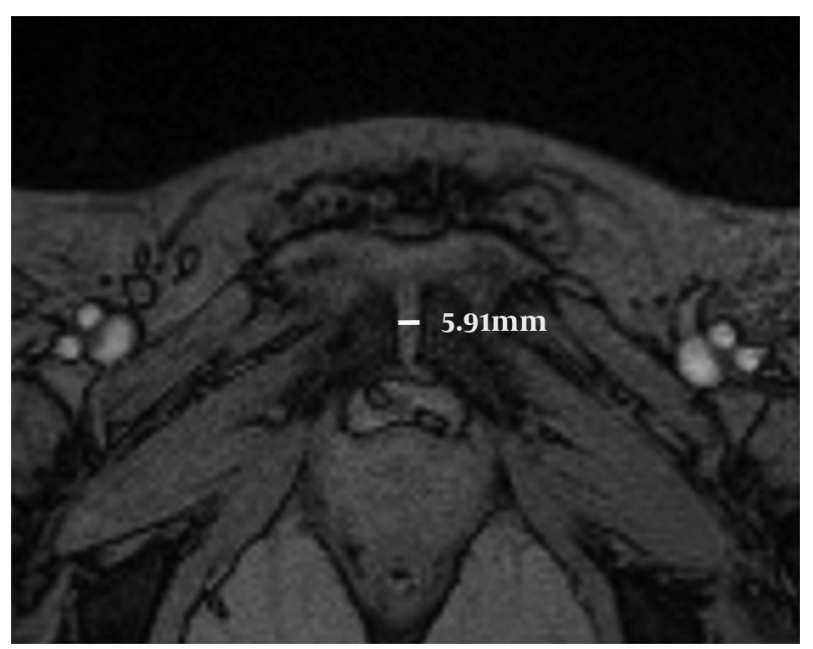

Figure 1. The pubic gap was measured on $\mathrm{TE}=11.83 \mathrm{~ms}$ images of one woman during her first pregnancy (28 years old, gestational age 35 weeks), the pubic symphyseal width was $5.91 \mathrm{~mm}$.

\subsection{Statistical Analysis}

Shapiro-Wilk test was used for the normality test to confirm that the data follow the normal distribution ( $\mathrm{P}>$ 0.05). The differences in age, height and pubic gap among the three groups were analyzed using ANOVA. Student's $t$ test was used to compare the gestational age between the first- and second pregnancies. To compare the $\mathrm{T} 2 *$ values in cartilage among the three groups, ANOVA and the least 

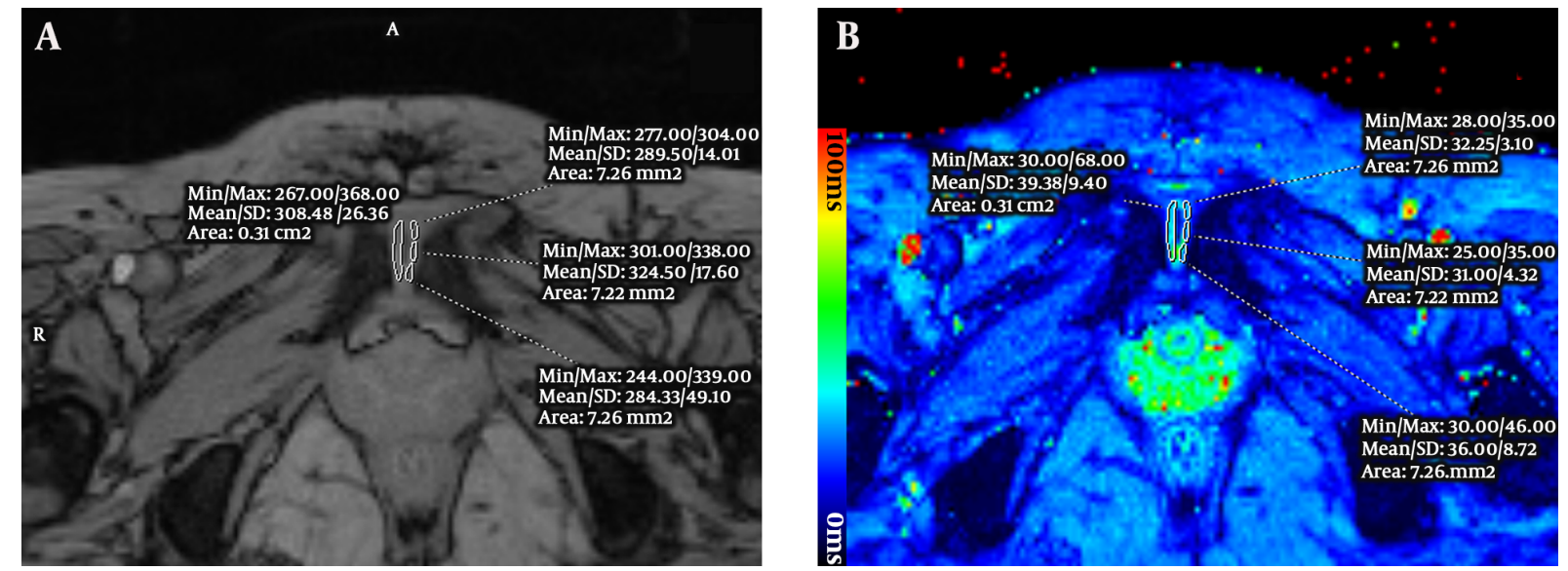

Figure 2. Eight regions of interest(ROIs)(left/right region and anterior/middle/posterior sub-regions of each side) were hand drawn on the cartilage of pubic symphysis based on TE $=11.83 \mathrm{~ms}$ image (A), then these ROIs were copied onto the T2* colored map (B). T2* values were measured.

significant difference (LSD) tests were performed. Correlation between the pubic symphyseal width and the T2* values of cartilage were analyzed using Pearson's correlation coefficient for each group. A P $<0.05$ was considered to be statistically significant. All the statistical analysis was conducted using SPSS Ver. 22.0 (IBM Corp., Armonk/NY, USA).

\section{Results}

Between March 2015 and December 2016, 116 pregnant women and 65 nulliparous volunteers were prospectively enrolled in this study. Seven pregnant women were excluded due to severe motion artifacts, one nulliparous woman did not finish the MRI scan because of claustrophobia. Sixty-one women during first pregnancy (mean age, $26.3 \pm 2.8$ years; range, 19 - 32 years; mean gestational age, $32.1 \pm 3.5$ weeks; range, 26 - 39 weeks), 48 women during second pregnancy (mean age, $28.5 \pm 3.8$ years; range, 23 38 years; mean gestational age, $31.6 \pm 3.5$ weeks; range, 25 37 weeks), and 64 nulliparous women of childbearing age (mean age, $25.7 \pm 2.0$ years; range, 21 - 32 years) were finally enrolled in this study. All subjects' information is listed in Table 1.

All data ed normal distribution $(\mathrm{P}>0.05)$. No statistical differences were noted among the three groups in age $(\mathrm{P}=0.123)$ or height distribution $(\mathrm{P}=0.687)$ (Table 1$)$. Moreover, no statistical differences were seen in the gestational age between the women during the first and second pregnancies.

The results revealed that the normal measurement of pubic symphyseal width for nulliparous women at child bearing age ranged from $3.3 \mathrm{~mm}$ to $9.6 \mathrm{~mm}$, women during first pregnancies ranged from $4.4 \mathrm{~mm}$ to $10.2 \mathrm{~mm}$, women during second pregnancies ranged from $4.6 \mathrm{~mm}$ to 11.8 $\mathrm{mm}$. The pubic symphyseal width increased significantly during late pregnancy $(\mathrm{P}<0.001)$; while, no differences were found between women during the first and second pregnancies $(\mathrm{P}=0.089)$.

All T2* values in different regions of cartilage between the pubic bones of the three groups are shown in Table 2 . The sub-region comparison of left /right regions showed that alterations of $\mathrm{T} 2^{*}$ values related to pregnancy were only seen in the posterior region (left $\mathrm{P}=0.004$; $\operatorname{right} \mathrm{P}=$ 0.002 ). It indicated that the differences in $\mathrm{T} 2^{*}$ values in the cartilage of pubic symphysis between pregnant women and nulliparous women were mainly driven by the posterior sub-region (Figure 3).

Compared with nulliparous women, the $\mathrm{T}^{*}$ values of cartilage increased significantly in pregnancy both on the left side $(\mathrm{P}=0.006)$ and right side $(\mathrm{P}=0.032)$; whereas, no differences were seen between the women during first and second pregnancy $(\mathrm{P}=0.143 ; \mathrm{P}=0.947)$ (Figures 3 and 4 ).

There was a positive linear correlation in women during first pregnancy $(\mathrm{r}=0.47, \mathrm{P}<0.001)$ between pubic symphyseal width and T2* values of cartilage (Figure 5 ), while there was no significant linear correlation in women during second pregnancy $(\mathrm{P}=0.216)$ and nulliparous women $(\mathrm{P}=0.635)$.

\section{Discussion}

T2 relaxation time reflects the ability of free water proton molecules to move and to exchange energy inside the cartilaginous matrix (17). T2 mapping has been widely used in the evaluation of articular cartilage $(2,3,6,21,22)$, and the numbers of studies have demonstrated it is a potential 


\begin{tabular}{|c|c|c|c|c|}
\hline & First pregnancy & Second pregnancy & Nulliparous controls & P-value \\
\hline Number & 61 & 48 & 64 & - \\
\hline Age, $y$ & $26.3 \pm 2.8$ & $28.5 \pm 3.8$ & $25.7 \pm 2.0$ & 0.123 \\
\hline Gestational age, wk & $32.1 \pm 3.5$ & $31.6 \pm 3.5$ & - & 0.338 \\
\hline Height, cm & $162.9 \pm 5.2$ & $163.7 \pm 4.5$ & $163.0 \pm 4.6$ & 0.687 \\
\hline Pubic width, mm & $6.2 \pm 1.2$ & $6.7 \pm 1.6$ & $4.8 \pm 1.1$ & 0.000 \\
\hline
\end{tabular}

${ }^{\mathrm{a}}$ Values are expressed as mean $\pm \mathrm{SD}$.

\begin{tabular}{|c|c|c|c|c|}
\hline \multirow{2}{*}{$\mathrm{T}^{*}$ values, $\mathrm{ms}$} & \multicolumn{3}{|c|}{ Groups } & \multirow{2}{*}{ P-value } \\
\hline & First pregnancy & Second pregnancy & Nulliparous controls & \\
\hline Left anterior & $18.62 \pm 4.19$ & $20.33 \pm 6.26$ & $17.93 \pm 4.58$ & 0.102 \\
\hline Left middle & $19.81 \pm 5.28$ & $20.79 \pm 7.91$ & $19.19 \pm 547$ & 0.708 \\
\hline Left posterior & $19.80 \pm 5.98$ & $20.64 \pm 6.92$ & $16.25 \pm 3.15$ & 0.013 \\
\hline Left region & $19.33 \pm 4.86$ & $20.73 \pm 5.86$ & $16.69 \pm 2.33$ & 0.006 \\
\hline Right anterior & $18.73 \pm 4.05$ & $17.47 \pm 4.91$ & $17.57 \pm 1.88$ & 0.235 \\
\hline Right middle & $19.31 \pm 7.79$ & $19.26 \pm 7.29$ & $18.62 \pm 3.97$ & 0.914 \\
\hline Right posterior & $19.81 \pm 5.29$ & $20.56 \pm 8.29$ & $15.61 \pm 3.52$ & 0.006 \\
\hline Right region & $19.19 \pm 4.62$ & $19.14 \pm 4.26$ & $16.52 \pm 3.30$ & 0.032 \\
\hline
\end{tabular}

${ }^{\mathrm{a}}$ Values are expressed as mean $\pm \mathrm{SD}$.

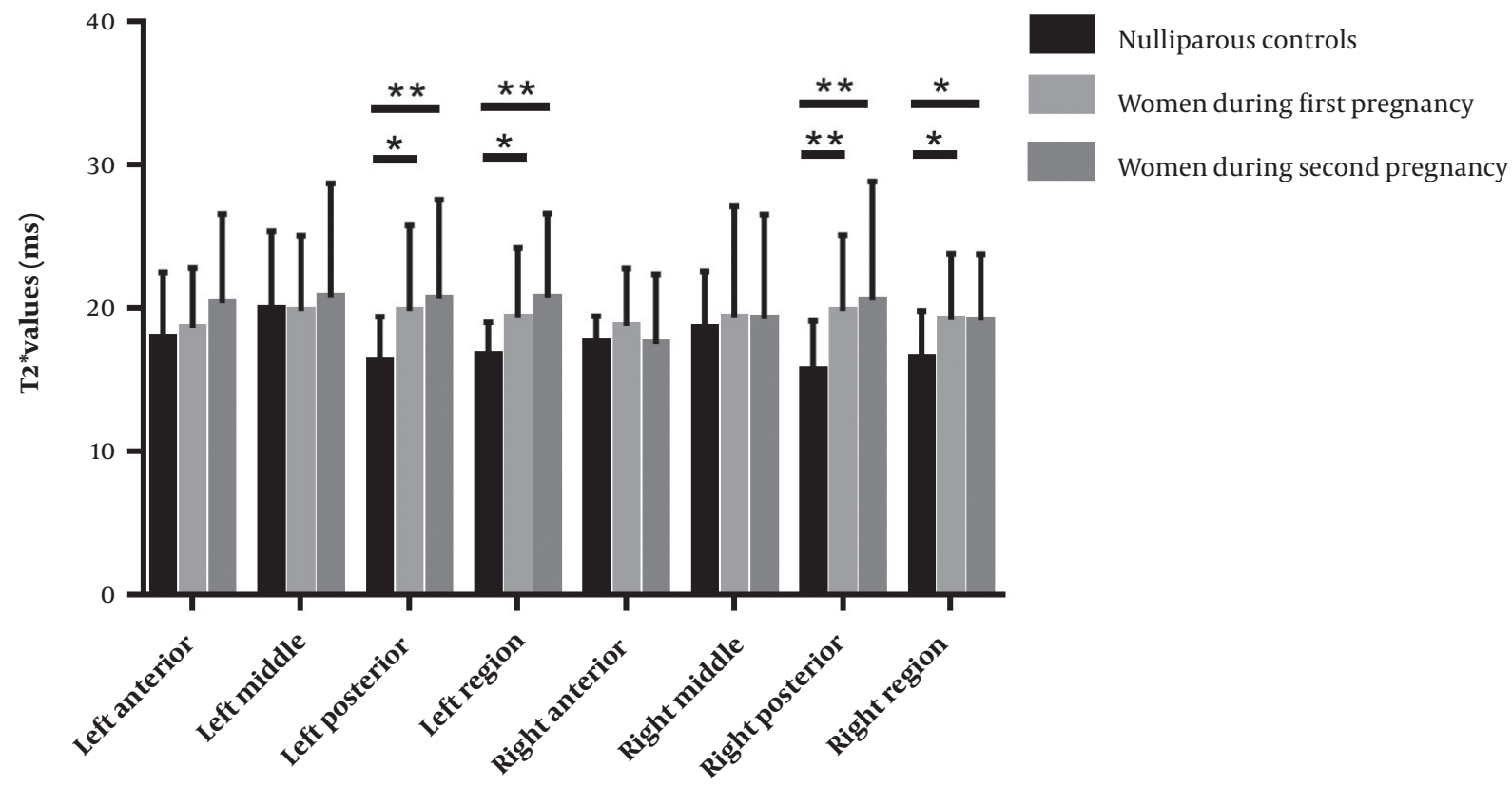

Figure 3. T2* values of cartilage between pubic bones in three groups. The differences between nulliparous women and first/second pregnant women were significant $(*$, means $\mathrm{P}<0.05$; ** means $\mathrm{P}<0.01$ ) in left/right regions and posterior sub-regions (left region $\mathrm{P}=0.028 / 0.001 ;$ left posterior $\mathrm{P}=0.015 / 0.004$; right region $\mathrm{P}=0.013 / 0.019$; right posterior $\mathrm{P}=0.007 / 0.002)$. 

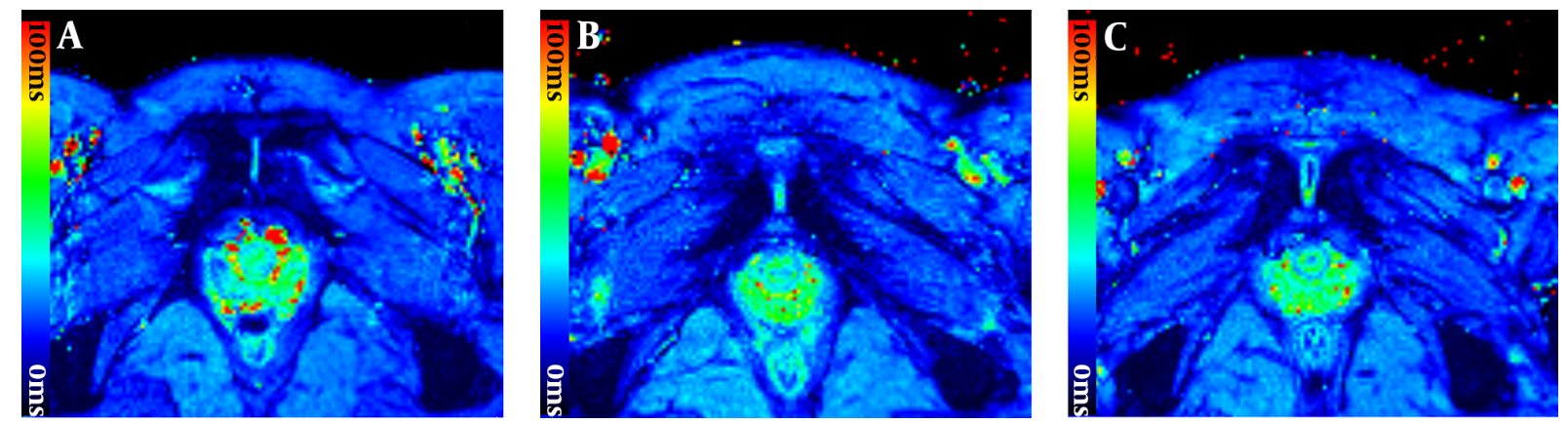

Figure 4. T2* maps of pubic symphysis show the color changes of cartilage during late pregnancy. A, A nulliparous woman, 29 years old; B, A woman during first pregnancy, 28 years old, gestational age 35 weeks; C, A woman during second pregnancy, 30 years old, gestational age 33 weeks.

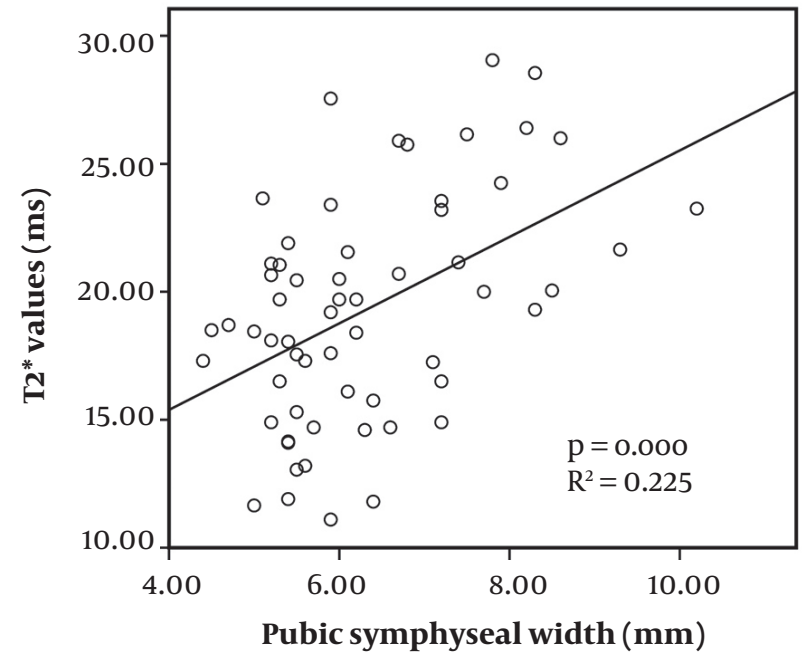

Figure 5. Correlation between $\mathrm{T} 2 *$ values and pubic symphyseal width in women during first pregnancy. T2*values were the average values of the left and right region of the cartilage. A positive linear relationship $(r=0.47, \mathrm{P}<0.001)$ is seen between them.

imaging biomarker for cartilage degeneration. T2* mapping is a technique similar to T2 mapping. It allows high image spatial resolution and shorter scan times $(17,20)$. Although T2* values have been widely used in many body regions, such as the intervertebral disc $(16,18,19,23,24)$, knee $(17,25,26)$, hip (27) or other articular cartilages (17), they have not been used previously for the assessment of pubic symphysis. So in this study, we chose $2^{*}$ mapping as the tool to assess cartilage with short acquisition time which is appropriate for pregnant women. The results indicated the $\mathrm{T} 2 *$ values of cartilage between pubic bones increased significantly during late pregnancy, and the differences were mainly driven by the posterior sub-region of cartilage; while, no differences were seen between women during first and second pregnancies. This study suggests that $2^{*}$ mapping is a sensitive quantitative method capable of detecting cartilage changes of the pubic symphysis during pregnancy.

The pubic symphysis is a secondary cartilaginous joint located between the left and right pubic bone, covered by a thin layer of hyaline cartilage attached to the fibrocartilage (1). In this study, the cartilage is mainly referred to fibrocartilage around the cleft in the center. $\mathrm{T} 2 *$ mapping is feasible to display the cartilage of pubic symphysis clearly, and the statistics results showed that T2* values of cartilage increase significantly during late pregnancy. The primary reason for this alteration is that the structure of pubic symphysis would change for the demand of childbirth. A hormone called relaxin released during pregnancy would increase the elasticity of tendons, ligaments, and muscles $(28,29)$, which induce the pressure from the fetus to easily widen the pubic gap and to stretch the cartilage between the pubic bones. Our results verified that the pubic symphyseal width increases from $4.8 \mathrm{~mm}$ to $6.2 / 6.7$ $\mathrm{mm}$ in first/second pregnancy. We further found a positive linear correlation between $\mathrm{T} 2{ }^{*}$ values of cartilage and pubic symphyseal width, which was only seen in women during first pregnancy. We assumed that the alterations of cartilage structure after first pregnancy were difficult to recover completely, so no significant linear correlation was seen in the women during second pregnancy.

The sub-region matrix comparison of cartilage demonstrated that the differences in $\mathrm{T} 2 *$ values between pregnant women and nulliparous controls were mainly driven by the posterior region of each side. There are four ligaments to maintain the stability of pubic symphysis, among which the posterior ligament is the thinnest one (1), so the posterior cartilage is the most prone to be stretched when the fetus presses the pubic symphysis.

Previous studies demonstrated a reduction in $\mathrm{T} 2 *$ val- 
ues with degeneration of articular cartilage (4, 18, 30-32). Our results are contrary to previous work investigating the tendency of cartilage $\mathrm{T} 2$ * values, the primary reason are the alteration of cartilage in this study is not related to aging, but pregnancy is in relatively short time. Other studies (3336) reported a similar conclusion that early degenerative changes have higher T2 values. The hormone effect and external force can increase the water contents of cartilage, which may be one of the reasons for increased $\mathrm{T} 2 *$ values during pregnancy.

The major limitation of our study was that there was no histological assessment of cartilage between pubic bones during pregnancy. Further investigation is needed to establish the relationship between $\mathrm{T} 2 *$ relaxation times and precise biochemical content, such as water, collagen, and proteoglycans.

In conclusion, $\mathrm{T} 2 *$ values of cartilage between pubic bones increased significantly during late pregnancy, which was mainly driven by the posterior sub-region, while no differences were seen between women during the first and second pregnancies. In summary, our study demonstrated the feasibility of using $\mathrm{T} 2 *$ to monitor the pregnancy-related fibrocartilage changes of the pubic symphysis.

\section{Acknowledgments}

We would like to thank all volunteers and patients for their participation.

\section{Footnotes}

Authors' Contributions: Contributed equally: TG and XZ. Study concept and design: TG and GBW. Analysis and interpretation of data: TG and XZ. Drafting of the manuscript: XZ. Critical revision of the manuscript for important intellectual content: TG, BBW, and SHY. Statistical analysis: SHY.

Conflict of Interests: All other authors declare no conflicts of interest.

Ethical Approval: This study was approved by Ethical Committee of Shandong Provincial Hospital (February 12th 2015 no.: 2015-016).

Funding/Support: This work was supported by Natural Science Foundation of Shandong (grant number: ZR2020QH267).

Informed Consent: All the participants provided written informed consent for this study.

\section{References}

1. Becker I, Woodley SJ, Stringer MD. The adult human pubic symphysis: a systematic review. J Anat. 2010;217(5):475-87. doi: 10.1111/j.1469-7580.2010.01300.x. [PubMed: 20840351]. [PubMed Central: PMC3035856].

2. Link TM, Neumann J, Li X. Prestructural cartilage assessment using MRI.J Magn Reson Imaging. 2017;45(4):949-65. doi:10.1002/jmri.25554. [PubMed: 28019053].

3. Chang EY, Ma Y, Du J. MR Parametric Mapping as a Biomarker of Early Joint Degeneration. Sports Health. 2016;8(5):405-11. doi 10.1177/1941738116661975. [PubMed: 27488128]. [PubMed Central: PMC5010134].

4. Guermazi A, Alizai H, Crema MD, Trattnig S, Regatte RR, Roemer FW Compositional MRI techniques for evaluation of cartilage degeneration in osteoarthritis. Osteoarthritis Cartilage. 2015;23(10):1639-53. doi: 10.1016/j.joca.2015.05.026. [PubMed: 26050864].

5. Hoessly ML, Wildi LM. Magnetic Resonance Imaging Findings in the Knee Before and After Long-Distance Running-Documentation of Irreversible Structural Damage? A Systematic Review. Am J Sports Med. 2017;45(5):1206-17. doi: 10.1177/0363546516656180. [PubMed: 27519676].

6. Le J, Peng Q, Sperling K. Biochemical magnetic resonance imaging of knee articular cartilage: T1rho and T2 mapping as cartilage degeneration biomarkers. Ann N Y Acad Sci. 2016;1383(1):34-42. doi: 10.1111/nyas.13189. [PubMed: 27472534].

7. Surowiec RK, Lucas EP, Ho CP. Quantitative MRI in the evaluation of articular cartilage health: reproducibility and variability with a focus on T2 mapping. Knee Surg Sports Traumatol Arthrosc. 2014;22(6):138595. doi: 10.1007/s00167-013-2714-6. [PubMed: 24170187].

8. Jazrawi LM, Alaia MJ, Chang G, Fitzgerald EF, Recht MP. Advances in magnetic resonance imaging of articular cartilage. J Am Acad Orthop Surg. 2011;19(7):420-9. doi: 10.5435/00124635-201107000-00005. [PubMed: 21724921].

9. Omar IM, Blount KJ. Magnetic Resonance Imaging of the Hip. Top Magn Reson Imaging. 2015;24(4):165-81. doi 10.1097/RMR.0000000000000057. [PubMed: 26244615].

10. Hebelka H, Miron A, Kasperska I, Brisby H, Lagerstrand K. Axial loading during MRI induces significant T2 value changes in vertebral endplates-a feasibility study on patients with low back pain. $J$ Orthop Surg Res. 2018;13(1):18. doi: 10.1186/s13018-018-0727-z. [PubMed: 29378613]. [PubMed Central: PMC5789539].

11. Hu J, Zhang Y, Duan C, Peng X, Hu P, Lu H. Feasibility study for evaluating early lumbar facet joint degeneration using axial T1 rho, T2 , and T2* mapping in cartilage. J Magn Reson Imaging. 2017;46(2):468-75. doi: 10.1002/jmri.25596. [PubMed: 28152249].

12. Ishikawa T, Watanabe A, Kamoda H, Miyagi M, Inoue G, Takahashi K, et al. Evaluation of Lumbar Intervertebral Disc Degeneration Using T1rho and T2 Magnetic Resonance Imaging in a Rabbit Disc Injury Model. Asian Spine J. 2018;12(2):317-24. doi: 10.4184/asj.2018.12.2.317. [PubMed: 29713414]. [PubMed Central: PMC5913024].

13. Messner A, Stelzeneder D, Trattnig S, Welsch GH, Schinhan M, Apprich S, et al. Does T2 mapping of the posterior annulus fibrosus indicate the presence of lumbar intervertebral disc herniation? A 3.0 Tesla magnetic resonance study. Eur Spine J. 2017;26(3):877-83. doi: 10.1007/s00586-016-4873-x. [PubMed: 27885476].

14. Paul CPL, Smit TH, de Graaf M, Holewijn RM, Bisschop A, van de Ven PM, et al. Quantitative MRI in early intervertebral disc degeneration: T1rho correlates better than T2 and ADC with biomechanics, histology and matrix content. PLoS One. 2018;13(1). e0191442. doi 10.1371/journal.pone.0191442. [PubMed: 29381716]. [PubMed Central: PMC5790235].

15. Pachowsky ML, Kleyer A, Wegener L, Langenbach A, Simon D, Janka $\mathrm{R}$, et al. Quantitative T2 Mapping Shows Increased Degeneration in Adjacent Intervertebral Discs Following Kyphoplasty. Cartilage. 2020;11(2):152-9. doi: 10.1177/1947603518758434. [PubMed: 29553284] [PubMed Central: PMC7097981]. 
16. Zhang Y, Hu J, Duan C, Hu P, Lu H, Peng X. Correlation study between facet joint cartilage and intervertebral discs in early lumbar vertebral degeneration using T2, T2* and T1rho mapping. PLoS One. 2017;12(6) e0178406. doi: 10.1371/journal.pone.0178406. [PubMed: 28570641]. [PubMed Central: PMC5453520].

17. Mamisch TC, Hughes T, Mosher TJ, Mueller C, Trattnig S, Boesch $\mathrm{C}$, et al. T2 star relaxation times for assessment of articular cartilage at 3 T: a feasibility study. Skeletal Radiol. 2012;41(3):287-92. doi: 10.1007/s00256-011-1171-x. [PubMed: 21499976].

18. Ellingson AM, Nagel TM, Polly DW, Ellermann J, Nuckley DJ. Quantitative $\mathrm{T}^{*}$ ( $\mathrm{T} 2$ star) relaxation times predict site specific proteoglycan content and residual mechanics of the intervertebral disc throughout degeneration. J Orthop Res. 2014;32(8):1083-9. doi: 10.1002/jor.22633. [PubMed: 24788830]. [PubMed Central: PMC4136382].

19. Ellingson AM, Mehta H, Polly DW, Ellermann J, Nuckley DJ. Disc degeneration assessed by quantitative $\mathrm{T} 2 *$ (T2 star) correlated with functional lumbar mechanics. Spine (Phila Pa 1976). 2013;38(24):E153340. doi: 10.1097/BRS.0b013e3182a59453. [PubMed: 23921323]. [PubMed Central: PMC3830665]

20. Krause FG, Klammer G, Benneker LM, Werlen S, Mamisch TC, Weber M. Biochemical T2* MR quantification of ankle arthrosis in pes cavovarus. J Orthop Res. 2010;28(12):1562-8. doi:10.1002/jor.21192. [PubMed: 20589929].

21. Hontoir F, Clegg P, Nisolle JF, Tew S, Vandeweerd JM. Magnetic resonance compositional imaging of articular cartilage: What can we expect in veterinary medicine? Vet J. 2015;205(1):11-20. doi: 10.1016/j.tvjl.2015.04.035. [PubMed: 26021889].

22. Niitsu M. [Articular cartilage MRI: Update]. Nihon Rinsho. 2016;74(6):924-30. [PubMed: 27311180].

23. Ellingson AM, Nuckley DJ. Altered helical axis patterns of the lumbar spine indicate increased instability with disc degeneration. J Biomech. 2015;48(2):361-9. doi: 10.1016/j.jbiomech.2014.11.010. [PubMed: 25481221]. [PubMed Central: PMC4286509].

24. Huang M, Guo Y, Ye Q, Chen L, Zhou K, Wang Q, et al. Correlation between $\mathrm{T} 2{ }^{*}$ (T2 star) relaxation time and cervical intervertebral disc degeneration: An observational study. Medicine (Baltimore). 2016;95(47) e4502. doi: 10.1097/MD.0000000000004502. [PubMed: 27893652]. [PubMed Central: PMC5134845].

25. Stelzeneder D, Shetty AA, Kim SJ, Trattnig S, Domayer SE, Shetty V, et al. Repair tissue quality after arthroscopic autologous collageninduced chondrogenesis (ACIC) assessed via T2* mapping. Skeletal Radiol. 2013;42(12):1657-64. doi: 10.1007/s00256-013-1708-2. [PubMed: 23990057].

26. Wilson KJ, Surowiec RK, Ho CP, Devitt BM, Fripp J, Smith WS, et al. Quantifiable Imaging Biomarkers for Evaluation of the Posterior Cruciate Ligament Using 3-T Magnetic Resonance Imaging: A Feasibility Study. Orthop J Sports Med. 2016;4(4):2325967116639040. doi:10.1177/2325967116639044. [PubMed: 27104206]. [PubMed Central PMC4827116].
27. Hesper T, Hosalkar HS, Schleich C, Antoch G, Welsch GH, Krauspe $\mathrm{R}$, et al. T2* Mapping for Hip Joint Cartilage Assessment: PreMRI Exercise and Time of Imaging Do Not Bias the T2* Measurement in Asymptomatic Volunteers. Cartilage. 2017;8(4):400-5. doi: 10.1177/1947603516665446. [PubMed: 28466650]. [PubMed Central: PMC5613890].

28. Samuel CS, Butkus A, Coghlan JP, Bateman JF. The effect of relaxin on collagen metabolism in the nonpregnant rat pubic symphysis: the influence of estrogen and progesterone in regulating relaxin activity. Endocrinology. 1996;137(9):3884-90. doi: 10.1210/endo.137.9.8756561. [PubMed: 8756561].

29. Pinheiro MC, Moraes SG, Battlehner CN, Caldini EG, Toledo OM, Joazeiro PP. Histochemical and ultrastructural study of collagen fibers in mouse pubic symphysis during late pregnancy. Micron. 2004;35(8):685-93. doi: 10.1016/j.micron.2004.04.007. [PubMed: 15288647].

30. Bittersohl B, Hosalkar HS, Miese FR, Schibensky J, Konig DP, Herten $\mathrm{M}$, et al. Zonal T2* and T1Gd assessment of knee joint cartilage in various histological grades of cartilage degeneration: an observational in vitro study. BMJ Open. 2015;5(2). e006895. doi: 10.1136/bmjopen-2014006895. [PubMed: 25667150]. [PubMed Central: PMC4322206].

31. Bittersohl B, Hosalkar HS, Hughes T, Kim YJ, Werlen S, Siebenrock KA, et al. Feasibility of $\mathrm{T}^{*}$ mapping for the evaluation of hip joint cartilage at 1.5T using a three-dimensional (3D), gradient-echo (GRE) sequence: a prospective study. Magn Reson Med. 2009;62(4):896-901. doi: 10.1002/mrm.22096. [PubMed: 19645008].

32. Miese FR, Zilkens C, Holstein A, Bittersohl B, Kropil P, Mamisch TC, et al. Assessment of early cartilage degeneration after slipped capital femoral epiphysis using $\mathrm{T} 2$ and $\mathrm{T} 2{ }^{*}$ mapping. Acta $\mathrm{Ra}$ diol. 2011;52(1):106-10. doi: 10.3109/02841851.2010.516015. [PubMed: 20954816].

33. Baum T, Joseph GB, Arulanandan A, Nardo L, Virayavanich W, Carballido-Gamio J, et al. Association of magnetic resonance imagingbased knee cartilage T2 measurements and focal knee lesions with knee pain: data from the Osteoarthritis Initiative. Arthritis Care Res (Hoboken). 2012;64(2):248-55. doi: 10.1002/acr.20672. [PubMed: 22012846]. [PubMed Central: PMC3267009].

34. Dunn TC, Lu Y, Jin H, Ries MD, Majumdar S. T2 relaxation time of cartilage at MR imaging: comparison with severity of knee osteoarthritis. Radiology. 2004;232(2):592-8. doi: 10.1148/radiol.2322030976. [PubMed: 15215540]. [PubMed Central: PMC4447089].

35. Joseph GB, Baum T, Alizai H, Carballido-Gamio J, Nardo L, Virayavanich $\mathrm{W}$, et al. Baseline mean and heterogeneity of MR cartilage T2 are associated with morphologic degeneration of cartilage, meniscus, and bone marrow over 3 years-data from the Osteoarthritis Initiative. Osteoarthritis Cartilage. 2012;20(7):727-35. doi:10.1016/j.joca.2012.04.003. [PubMed: 22503812]. [PubMed Central: PMC3595156].

36. Mosher TJ, Dardzinski BJ. Cartilage MRI T2 relaxation time mapping: overview and applications. Semin Musculoskelet Radiol. 2004;8(4):35568. doi: 10.1055/s-2004-861764. [PubMed: 15643574]. 